\title{
LA MODERNIZACIÓN DEL SISTEMA EDUCATIVO EN FRANCIA: LA NUEVA GESTIÓN PÚBLICA ENTRE LA AFIRMACIÓN DEL ESTADO Y LA GOBERNANZA DESCENTRALIZADA
}

\author{
JeAn-Louis Derouet \\ ROMUALD Normand ${ }^{* *}$ \\ RAMON GP PACHECO
}

\begin{abstract}
RESUMEN: La historia de la administración pública francesa en educación se encuentra sólidamente vinculada al legado napoleónico y al nacimiento de la Tercera República. La importancia del legalismo presenta un impacto en la acción de los diseñadores de políticas, inspectores y directores de los diferentes niveles jerárquicos, situándoles en posiciones de mando, autoridad y subordinación. Debido al peso de los servicios públicos y del funcionariado en Francia, es principalmente el Estado quien se está viendo transformado con algunos efectos sobre el sector de la educación. Este orden burocrático es hoy día desafiado por la implementación de la Nueva Gestión Pública o New Public Management (NPM). La ley para la modernización de las finanzas públicas ha reorganizado el gasto público en educación de acuerdo a amplios programas que implementan los 3 principios del NPM: economía, eficiencia, efectividad. Sin embargo, Francia ha desarrollado una rendición de cuentas carente de mercado educativo. Se trata además de un NPM sin demasiada descentralización y flexibilidad. Mientras otros sectores implicados en las políticas públicas, como el sanitario, han conocido ya importantes cambios en su organización y condiciones de trabajo, el educativo parece adentrarse en una nueva etapa marcada por la reestructuración de las profesiones relacionadas con la enseñanza. La mejora de la comprensión del proyecto de modernización y NPM en el sector de la educación pasa por observar su relación con algunas transformaciones importantes y globales dentro del Estado central. La politización de diseñadores de políticas de alto rango, diversificación de servicios y responsabilidades, individualización y movilidad, o pago por actividad puesta en práctica, son algunos de los principales
\end{abstract}

\footnotetext{
* École Normale Supérieure de Lyon, Institut Français de l'Éducation, Laboratoire Triangle. Lyon, France. E-mail de contacto: jeanlouis.derouet@ens-lyon.fr.

** Université de Strasbourg, Faculté des sciences sociales, Sociétés, Acteurs et Gouvernement en Europe (Sage). Strasbourg, France.
} 
componentes de la desregulación del Estado que presenta impacto sobre los profesionales desde formas más sutiles e invisibles.

Palabras clave: Nueva gestión pública; Francia; Educación; Eficiencia; Efectividad; Accountability.

\section{THE MODERNIZATION OF THE EDUCATION SYSTEM IN FRANCE: THE NEW PUBLIC MANAGEMENT BETWEEN STATE AFFIRMATION AND A DECENTRALIZED GOVERNANCE}

ABSTRACT: The history of the French public administration in education is strongly linked to Napoleon's legacy and the birth of the Third Republic. The importance of legalism has had an impact on the action of policy-makers, inspectors and principals at different hierarchical levels, and it places them in positions of command, authority and subordination. Because of the weight of public services in France, and the weight of public servants, it is mainly the State which is transformed with some effects on the sector of education. This bureaucratic order is today challenged by the implementation of the New Public Management. The act of modernization of public finances has reorganized public expenditures in education according to large programs implementing the 3 principles of NPM: economy, efficiency, effectiveness. However, France has developed a soft accountability without a school market. It is also a NPM without much decentralization and flexibility. However, the next step could be the restructuring of the professions in education while other sectors of public policies, such as health, have already known important changes in their organizations and conditions of work. To better understand the project of modernization and New Public Management in the education sector, it is also important to relate it to some important and global transformations within the central State. Politicization of high rank policy makers, diversification of services and responsibilities, individualisation and mobility, pay-related performance are some main components of the deregulation of the State which impacts professionals in more subtle and invisible ways.

Keywords: New public management; France; Education; Economy; Efficiency; Effectiveness; Accountability.

\section{LA MODERNISATION DU SYSTÈME ÉDUCATIF EN FRANCE: LE NOUVELLE GESTION PUBLIQUE ENTRE LA DÉCLARATION DU GOUVERNEMENT ET LA GOUVERNANCE DÉCENTRALISÉE}

RÉSUMÉ: L'histoire de l'administration publique française en éducation est étroitement liée à l'héritage napoléonien et à la naissance de la Troisième République. L'importance du légalisme a eu un impact sur l'action des décideurs politiques, inspecteurs et chefs d'établissement à différents niveaux hiérarchiques, et il les place dans des positions de commandement, d'autorité et de subordination. En raison du poids des services publics en France, et de celui des fonctionnaires, c'est 
principalement l'Etat qui connaît des transformations avec des effets sur le secteur de l'éducation. Cet ordre bureaucratique est aujourd'hui mis à l'épreuve par la mise en œuvre de la Nouvelle Gestion Publique ou New Public Management (NPM). La Loi Organique relative aux Lois de Finances (LOLF) a réorganisé les dépenses publiques en éducation selon les grands programmes en prenant les trois principes de la NGP: coût, efficience, efficacité. Néanmoins, la France a développé une obligation de résultats en douceur sans marché scolaire. C'est aussi une NGP sans beaucoup de décentralisation et de flexibilité. Malgré tout, la prochaine étape pourrait être la restructuration des professions de l'éducation alors que d'autres secteurs des politiques publiques, comme la santé, ont déjà connu d'importants changements dans leurs organisations et conditions de travail. Afin de mieux comprendre le projet de modernisation et de la Nouvelle Gestion Publique dans le secteur éducatif, il est aussi important de la relier aux transformations globales à l'intérieur de l'Etat. La politisation des responsables, la diversification des services et des responsabilités, l'individualisation et la mobilité, la rémunération à la performance sont les principales composantes de la dérégulation de l'État qui impact de manière plus subtile et invisible sur les professionnels.

Mots-clés: Nouvelle gestion publique; France; Éducation; Santé; Efficacité; Décentralisation; Économie.

\section{Introducción}

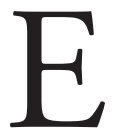

n el marco de la globalización, el New Public Management (NPM) a la francesa en educación parece escapar de toda influencia en la circulación de modelos. Éste, por un lado, mantiene las distancias respecto al modelo anglosajón, del cual rechaza los principios neoliberales, al tiempo que se encuentra en la búsqueda infructuosa de una gobernanza local, lo que lo diferencia del modelo escandinavo o germánico, en los cuales la descentralización se encuentra más avanzada. Por otro, se construye en autorreferencia a la tradición republicana y napoleónica que ha marcado el surgimiento del estado burocrático moderno al cual se encuentra asociado el proyecto político de escuela moderna. Ello explica la importancia de la historia política y la herencia cultural del sistema educativo en la comprensión de las tentativas de modernización puestas en práctica en el curso de los últimos años. Si lo que se persigue es caracterizar el desarrollo del NPM en Francia de acuerdo a las categorías sociológicas puestas en evidencia por Max Weber, es posible la descripción de esas transformaciones como acción racional en valores y no en finalidades, dado que la búsqueda de la eficacia es con frecuencia secundaria en las formas de justificación de las reformas educativas desde la época napoleónica. 
La administración educativa francesa se ha mostrado siempre orgullosa de sus tradiciones, articulándose éstas de acuerdo a dos hitos, uno de partida, que se remonta a la Ilustración, y un segundo de estabilización, que tiene lugar durante el Imperio Napoleónico. Un buen número de planes educativos fue publicado durante la segunda mitad del siglo XVIII, afirmando el siguiente principio: la educación es asunto del Estado; no concierne, pues, a familias, comunidades y mucho menos congregaciones religiosas. A nivel local, todo ello cultivó una gran desconfianza que acabó siendo reforzada por los planes estatales que tuvieron lugar en la década de los 60 del siglo XX. Estos últimos definían una zona escolar de captación para la matriculación del alumnado en función de la localización de sus hogares. Un interés de corte estatista que inspiró también la definición de un currículum centrado en las disciplinas académicas y el acceso al universalismo. Por un lado, esta tradición republicana francesa se encuentra sustentada por cuerpos profesionales clave como la Inspection Générale, o directamente incorporada en la Agrégation (un examen especial de selección para obtener el reconocimiento de profesor de alto rango de la educación secundaria francesa); por otro, ha penetrado tanto en la cultura de la enseñanza profesional como en el poder compensatorio de los sindicatos. Todo proyecto de modernización escolar hubo de ser ajustado y adaptado a dicho marco: ese es el caso de la escuela comprensiva francesa, pero también el de la implementación del NPM.

El sistema educativo francés es principalmente público. Un sistema privado, católico en mucha mayor medida, acoge en torno al 17\% del alumnado. Desde la separación de Iglesia y Estado (1905) la educación privada dejó de ser subsidiada por más de medio siglo. Sin embargo, a principios del régimen político de la 5a República, la ley Debré (1959) establece un compromiso: una educación de tipo privado y sujeta a contrato será subsidiada, pero se hará respetando las regulaciones del Estado, en concreto desde la impartición de un currículum idéntico, la sujeción a las mismas inspecciones y una igual formación del profesorado.

Desde mediados de los 70 hasta los años 80, el sistema francés de escuela comprensiva (college unique) fue implementado por los distintos gobiernos tanto de derechas como de izquierdas. Esta política representaba una promesa de democratización para muchos educadores y padres, pero no satisfacía sus expectativas y esperanzas. (DEROUET, 1992) Ello desembocó en una crisis de confianza en el sistema educativo, al tiempo que algunos intelectuales vinculados tanto a la derecha como a la izquierda denunciaban la "falsa democratización" de la educación secundaria que conducía, para muchos de ellos, a la "derrota de la reflexión”. Por otro lado, el sistema fue desafiado por las quejas frente al reconocimiento de las diferencias étnicas y religiosas afirmadas durante los últimos ańos del siglo XX. (HONNETH, 2000; FRASER, 2013) La desconfianza de la tradición republicana francesa hacia las comunidades y el multiculturalismo permanece como obstáculo a la comprensión intelectual del problema. En un período en el que la 
Unión Europea apuesta por la inclusión social de las minorías, algunos sectores de la sociedad francesa se ven tentados a volver a definiciones más tradicionales de la república y al laicismo. Estas herencias serán descritas en la primera parte de este capítulo.

El clima de pesimismo asociado al fracaso del sistema basado en la escuela comprensiva facilitó la introducción por parte de organizaciones internacionales de algunas recomendaciones relacionadas con la rendición de cuentas o accountability. (NORMAND, 2011) Sin embargo, tanto la izquierda como la derecha se mantienen hostiles a la introducción de ideas provenientes del mercado en educación. Además, ambas presentan dudas en cuanto a gestión y "managerialización". De forma tradicional, la izquierda se encuentra unida al funcionariado, desconfiando de los discursos empresarial y gerencial. Existe, en cambio, "otra" izquierda que está promoviendo algunas nuevas ideas sobre gobernanza, descentralización, democracia local y disminución de la intervención estatal. Ello, sin embargo, no ha llevado a los diseñadores de políticas franceses a convertirse al liberalismo y a las políticas de libre mercado: algunos de ellos están solamente reaccionando contra el estado burocrático, reclamando más eficiencia y calidad. En general, el sistema educativo francés ha incluido algunos principios del New Public Management en su tradición burocrática.

Todas estas cuestiones vienen referidas a diferentes valores y sirven para entrelazar las agendas políticas nacional e internacional. Se torna complicado, pues, caracterizar los distintos legados y cambios desde los últimos cincuenta ańos. Sin embargo, es posible proporcionar el siguiente análisis en relación a la introducción del New Public Management en la administración francesa: un periodo de relativa apertura desde inicios de los 80, marcado por una ley general descentralizadora votada en 1981, y por una nueva definición de justicia importada de los ejemplos británicos. (DEROUET; DEROUET-BESSON, 2008) Este movimiento se encuentra en la raíz tanto de la filosofía de zonas de educación prioritaria como de las políticas de autonomía escolar, siendo ampliado tras la primera Conferencia de Lisboa (2000) como consecuencia de la instigación sufrida por Francia para que ésta tomara en cuenta las recomendaciones europeas clave. La primera medida ligada a esta nueva directriz fue la LOLF, o Ley Orgánica relativa a las Leyes de Finanzas (Loi Organique relative aux Lois de Finances), aprobada por unanimidad por el Parlamento en 2001, la cual proponía una nueva organización de los servicios públicos basada en la accountability. La segunda dirección propuesta es un marco de competencias que reemplazaría la estructuración del currículum por disciplinas (Ley 2005). Esta concepción se encuentra alejada de la tradición francesa y su impacto real entre profesionales puede ser cuestionado, pero las competencias forman parte hoy día de la cultura política y profesional. Comparativamente, los últimos ańos invitan a pensar en un cierre de la identidad nacional. La concepción tradicional de la república se encuentra amenazada y el partido socialista 
gobernante desde el año 2012 ha vuelto a sus principios fundamentales: servicio público, centralización, laicismo.

\section{Legados, narrativas y políticas de modernización}

Las últimas décadas han sido testigo de una intensa actividad legislativa. La Ley de Modernización de la Escuela de 1975 creó el sistema de escuela comprensiva (collège unique). La noción "plan de desarrollo escolar" fue consagrada por la Ley 1989, y ha servido desde entonces para vertebrar la regulación del sistema educativo. Se trata de una ley "paraguas" que fija los principios clave al tiempo que dota de cierta autonomía al nivel local. En cualquier caso, este tipo de compromiso, inspirado en la educación progresiva y en la idea de "situar al alumno o alumna en el centro del sistema educativo", no ha sido realmente entendido, habiendo sido incluso rechazado por la mayor parte de la comunidad educativa, para quien la transmisión del conocimiento, la enseñanza y no el aprendizaje, tiene que ser la principal preocupación del sistema. Finalmente, la sociedad en su conjunto no solo quedó decepcionada por los pobres resultados de las escuelas comprensivas en la reducción de las desigualdades, sino que comenzó a preocuparse por los efectos de la escolarización extensiva: el sistema educativo no logró traer los avances sociales que se esperaban. Además, se creó una atmósfera de desencanto en relación al logro escolar del alumnado. Las primeras publicaciones arrojadas por encuestas internacionales no eran tranquilizadoras para los pesimistas. El vínculo republicano entre escuela y sociedad se había roto.

Más allá de estas dudas de la sociedad francesa, la Conferencia de Lisboa (2000) de la Unión Europea introdujo algunos elementos que fueron implementados en los objetivos de la Ley 2005. Desde el área de la jurisprudencia se introdujo un marco de habilidades básicas inspirado por el marco europeo de competencias clave y definido por la Comisión Europea para la Educación Permanente. Cuando la izquierda obtuvo el poder en 2012, promulgó una ley para la refundación del sistema educativo que sirvió para expresar la sensación de deriva de la sociedad francesa en relación a su educación, así como el derecho de retomar la tradición neokantiana de los fundadores de la escuela republicana de principios de los 80 del siglo XIX. La pasión laicista, que había perdido fuelle a raíz de la decadencia de la Iglesia Católica, ha retomado poder frente al fundamentalismo islámico.

Esta actividad legislativa fue apoyada por la creación de herramientas para la policy, las cuales se encargaron de su implementación. 


\section{Descentralización y Autonomía de los Centros Educativos}

El intento de descentralización sucedió a una reflexión posterior al movimiento de 1968 en torno a la posibilidad de que los centros educativos se convirtieran en unidades de gestión escolar, al tiempo que el sistema de escuela centralizada, con su millón de funcionarios, era con frecuencia comparado con el Ejército Rojo. A comienzo de la década de los 70 se experimentó un considerable número de medidas pero la Ley 1975 acabó con esta tendencia y Francia volvió a la tradición del plan estatal. Francia puso en marcha el sistema de escuela comprensiva más tarde que otros países miembros de la OCDE. La noción de autonomía escolar, la cual ha sido conceptualizada desde una perspectiva pedagógica, asumió entonces un sentido gerencial. La nueva idea de plan de desarrollo escolar fue introducida en 1982 como un experimento durante la reforma de las escuelas junior. En 1984 un decreto de la ley de descentralización general proporcionó a cada centro de enseñanza secundaria el estatus de Escuela Pública Local, otorgándose la posibilidad a la dirección de los mismos de definir su propio plan de desarrollo escolar. La izquierda añadió un objetivo social: adaptar los métodos de aprendizaje a las necesidades del alumnado de cara a prevenir desigualdades escolares. Sin embargo, una cierta visión de gestión permaneció, siendo inspirada por las ideas del sociólogo Michel Crozier. El título de uno de sus libros resume su pensamiento: "Estado Modesto, Estado Moderno" (1986).

De forma paralela a la extensión de la noción de un plan de desarrollo escolar a lo largo de todo el sistema educativo, la Ley 1989 se reveló como el pilar de las nuevas regulaciones. Ésta propuso el establecimiento de una sucesión de contratos a nivel individual y moral entre el alumno, la escuela y su familia, todo ello sin renunciar al ya mencionado concepto de zona escolar de captación. Sin embargo, la ley introdujo, aunque encuadradas y limitadas, algunas posibilidades de elección educativa para familias que no aceptaban el proyecto basado en la escuela, lo que significó el reconocimiento de los derechos para las familias que no se dirigían a un sistema basado en el mercado. Otra de las limitaciones de esta autonomía fue el "intocable" currículum nacional. En consecuencia, la autonomía quedó rápidamente restringida a una angosta y local gestión carente de medios flexibles para el cumplimiento de los objetivos nacionales.

La Ley 2005 intentó resucitar el principio de autonomía escolar a través de una cautelosa concepción liberal. La medida principal fue la creación de una "dirección pedagógica": los sindicatos se negaron a permitir que las cuestiones relacionadas con la enseñanza fueran tratadas desde el nivel de dirección administrativa, dado que muchos de los miembros de la dirección no eran competentes en el campo. Pero la dirección pedagógica, que sólo incluía a miembros del profesorado, pudo finalmente gestionar el currículum nacional así como las condiciones de enseñanza local. Sin embargo, su implementación fue larga y complicada, arrojan- 
do efectos decepcionantes. La ley 2005 asumió igualmente las recomendaciones de las organizaciones internacionales en relación a la diversificación del currículum escolar como medio para promover la efectividad y la equidad. El artículo 34 de la Ley contemplaba varias posibilidades para la innovación, permitiendo a los centros obtener mayor libertad fuera de las regulaciones nacionales. Esta medida logró ayudar a algunos centros a construir una identidad específica; sin embargo ni la elección de estos ni los planes de desarrollo escolar, con la notable excepción de unos pocos, se revelaron novedosos o creativos. Siguiendo la misma lógica, en 2007, el Ministerio anunció una mayor flexibilidad en la política de la zona escolar de captación, siendo su abolición programada en 2010. Tras un acalorado debate, algunos cambios fueron realizados a esta declaración. Las autoridades locales, las cuales se posicionaban mayoritariamente contra la libre elección de centro, no llevaron a cabo esas instrucciones, y ello ha seguido limitando las posibilidades de aquellas familias que desean mantenerse fuera de su ámbito. La izquierda, de vuelta en el poder, anuló esa política y reforzó aquélla de la zona escolar de captación.

\section{Desarrollo de la evaluación}

El período desde mediados de los 1980 hasta mediados de los 1990 viene caracterizado por la implantación de un sistema de evaluación. Es el resultado de una larga historia. Durante la década de los 1970, el antiguo sistema de estadística administrativa evolucionó hacia nuevas misiones y objetivos, en lo que supuso el desarrollo de un sistema de evaluación. En 1986 este servicio administrativo pasó a formar parte de la dirección ministerial: la DEP, o Dirección de la Evaluación y la Prospectiva (Direction de l'Évaluation et de la Prospective). Los sucesivos responsables de la Direction han compartido el mismo pensamiento en torno a las encuestas de profundidad a gran escala así como a la cultura de las estadísticas del Estado. La DEP acumulaba en su seno importantes responsabilidades. Servía de bálsamo para quienes temían que la autonomía de los centros desembocara en una pérdida de control en la conducción del sistema educativo; la ley habilitaba el sistema de evaluación para prevenir "alguna posible deriva". En respuesta a las preocupaciones de la sociedad en torno a la calidad del aprendizaje, la DEP también tenía la responsabilidad de evaluar regularmente las habilidades del alumnado en diferentes etapas del sistema educativo. Todas esas misiones se encontraban inscritas en una particular concepción de política pedagógica: el objetivo no era otro que diseñar herramientas para el Estado a través de indicadores construidos desde una perspectiva nacional, los cuales tendrían en cuenta la diversidad de las prácticas locales. 
En nombre de su misión predictiva, la DEP ofertó varias convocatorias para investigación en educación. La primera, a finales de los 1980, suponía la vuelta a las inversiones en educación, mientras sutilmente introducía los principios de la accountability francesa. El retorno de la inversión no era únicamente medido desde la puesta en práctica, sino a través de la reducción de la desigualdad de oportunidades. La segunda misión se ocupaba de la inversión educativa de las familias. Esta convocatoria seguía la estela de un movimiento emergente sobre consumo escolar y nuevas opciones para escuelas privadas, en el que se subrayaba la realidad e importancia de la violencia escolar. Todos los resultados de investigación arrojados por los proyectos seleccionados eran presentados en informes de la Dirección, siendo difundidos y sintetizados por prensa y medios. Tras este próspero período, las misiones de la DEP fueron revisadas y reducidas para finales de los 1990: habían alcanzado mucha influencia en comparación con otras unidades directivas, incluso sobre el propio Ministro, y debían reintegrarse en expedientes y clasificaciones. El debate fue arduo: ¡era normal que la evaluación fuera conducida por un Ministerio que designaba e implementaba las policies educativas? Varias reflexiones se inspiraron en ejemplos de países escandinavos, donde las instituciones evaluadoras se encuentran bajo control del Parlamento. Pero ese concepto no es plenamente familiar para los policymakers franceses. Incluso el término "agencialidad" es por éstos considerado demasiado liberal, prefiriendo "altos consejos" que mantienen una importante dependencia del Estado. Un Consejo Nacional para la Evaluación del Sistema Educativo, conocido por sus siglas CNESCO (Conseil National d'Évaluation du Système Scolaire), fue creado en 2014. Un académico fue designado Presidente del Consejo por el Ministro, aunque todos los recursos son provistos por departamentos del Ministerio. Además, este nuevo consejo no abolió los anteriores: la DEP permanece activa, mientras que la Inspección se encuentra todavía a cargo de la evaluación de profesores y centros.

Esta situación puede ser considerada como emblemática. Los principios del New Public Management han sido afirmados, y ello no es una sentencia puramente retórica. Se ha creado un espacio para una importante actividad legisladora y reguladora: Francia ha adoptado progresivamente las recomendaciones europeas. Pero en base a una extraña composición, estos principios han sido incluidos en la mentalidad administrativa francesa, la cual ha reformulado las cuestiones clave. Esta es la razón por la que algunos politólogos llaman a esta evolución "dependencia del camino" o "hibridación de políticas" en un contexto nacional. Este proceso limita o incluso neutraliza el impacto de las recomendaciones internacionales de la OCDE y la Comisión Europea. Desde esta perspectiva, es posible ilustrar las paradojas de esta modernización y examinar cómo el New Public Management ha sido implementado en diferentes áreas con algunos ejemplos de préstamos de políticas desde otros países y organizaciones internacionales. 


\section{Más allá de los legados y el reformismo: las paradojas del New Public Management francés en Educación}

Si la planificación, a través de la acción del Comité de Planificación, fue considerada por mucho tiempo como palanca para conciliar los objetivos de igualdad de oportunidades y desarrollo económico, la crisis económica y el fracaso de la escuela comprensiva forzó al estado educativo a un cambio de políticas. A principios de los 80 , como describíamos en la primera parte, la orientación era la preocupación principal de los diseñadores de políticas, emergiendo la evaluación como nueva herramienta para la gobernanza del sistema educativo. Ello explica el desarrollo de la primera evaluación nacional y la creación de la Dirección de la Evaluación y la Prospectiva por parte del Ministerio de Educación Nacional.

Tras las leyes descentralizadoras, el New Public Management francés ha equivalido a un proyecto de modernización educativa, al tiempo que, contrariamente a lo sucedido en otros países, ha resistido firmemente a tendencias de mercado y privatización. (POLLITT, 1990; POLLITT; BOUCKAERT, 2004) Como poníamos de manifiesto en la primera parte, el legado republicano es sólo una primera explicación: el sistema escolar republicano siempre mostró entusiasmo en hacer retroceder los intereses privados conforme la educación iba siendo progresivamente unificada como servicio público. Por tanto, la reforma NPM representa un compromiso entre tradición y modernización, lo que facilita la emergencia de numerosas paradojas como la de la pasada década, momento en que la política educativa francesa ha comenzado a volverse cada vez más permeable a los efectos de la globalización y la europeización. (HOOD, 1991; HOOD; PETERS, 2004)

\section{Evaluación respecto a una lógica burocrática}

La creación de la Dirección de la Evaluación y la Prospectiva representa un buen ejemplo de este tipo de compromiso francés. Encontrándose inspirada en la School Effectiveness Unit creada por el Departamento de Educación del Reino Unido, fue inicialmente concebida como un instrumento de planificación para predecir la matriculación de alumnos. Ello tuvo lugar tras el anuncio por parte del Ministro socialista Jean-Pierre Chevènement del objetivo de que "[...] el 80\% de una misma generación obtenga el bachillerato en 2000 [...]". Pero ello era también el resultado de una presencia de Francia en comités internacionales de expertos y a su implicación, junto a los Estados Unidos y la OCDE, para, desde éstos, diseñar indicadores internacionales en educación. Si bien la lógica de evaluación había penetrado progresivamente en el sistema educativo francés, no se ocupaba de evaluar su calidad o efectividad, al menos en un principio. Las evaluaciones 
nacionales, como indicadores para los centros, eran herramientas diseñadas para medir la desigualdad en los resultados del alumnado, siendo presentadas como un medio para reducir dichas desigualdades, así como para democratizar el acceso a la educación. Los objetivos del Ministerio no eran promover la libre elección de centro y la lógica del mercado sino combatir las clasificaciones publicadas por la prensa, las cuales dificultaban la evaluación justa de las características sociales y el mérito de cada centro. Hoy día los exámenes son todavía formativos y no sumativos: sirven al profesorado para mejorar sus prácticas de enseñanza pero no son utilizados para seleccionar al alumnado.

Es durante los años 90 cuando la evaluación comenzó a ser considerada como herramienta para la medida de la efectividad y calidad del sistema educativo. Mientras tanto, un nuevo paradigma emergía. Claude Thélot, quien jugó un papel como director de la DEP, fue el conductor de esta transformación. (THÉLOT, 1993) La evaluación abrazaba los principios del New Public Management (economía, eficiencia, efectividad) mientras se creaba un Alto Consejo para la Evaluación de la Educación (Haut Conseil de l'Évaluation de l'École). Éste se convierte rápidamente en un espacio de reflexión para expertos y responsables de políticas educativas. El Alto Consejo emitía informes que demandaban que el sistema francés de evaluación se alineara con las encuestas internacionales conducidas por la OCDE, especialmente con el informe PISA. (HENRY; ALII, 2001) Igualmente, el Alto Consejo promovía la idea de un marco de competencias, tras una extendida consulta nacional titulada "El Gran Debate sobre Centros" basada en datos y cuestiones preparados por la DEP con el apoyo de una agencia de consultoría. Posteriormente, Francia se unió al Comité Europeo para Indicadores y Estándares para participar en la construcción de los indicadores del Método Abierto de Coordinación. El informe PISA se ha convertido progresivamente en un punto de referencia para los diseñadores de políticas, destacando Finlandia como ejemplo de reforma educativa implementada con éxito.

En Francia, la educación es, por un lado, un servicio público y, por otro, una administración individual del Estado. (DEROUET, 2000) Ésta se encuentra, por tanto, directamente sujeta a las reformas promulgadas por el Estado. Como se ha visto, la evaluación se ha convertido en un componente fundamental para las acciones del Estado canalizadas por la Ley Institucional relativa al paquete de Leyes Financieras de 2001 (Loi d'Organisation des Lois de Finance, también llamada LOLF). La citada Ley institucionalizaba nuevas regulaciones para el gasto público a través de programas nacionales y objetivos que tenían que ser evaluados. (CYTERMANN, 2009) Por tanto, cada administración individual y departamento del Estado tenía que rendir cuentas. Pero la rendición de cuentas o accountability en educación resulta muy administrativa, se encuentra muy influida por la dimensión financiera e, incluso, cuando incorpora a sus indicadores los resultados de los exámenes que realiza el alumnado, al contrario de lo que ocurre en Inglaterra, no 
presiona a los centros en lo que respecta al rendimiento. (MAHONY; HEXTALL, 2000; GLEESON; HUSBANDS, 2001) De hecho, ningún sistema de información o herramienta digital para la evaluación ha sido desarrollado para hacer al profesorado responsable en mayor medida. La LOLF resultó muy burocrática, y ha servido principalmente para justificar los procesos de toma de decisiones para la reducción de presupuestos así como procesos para la aplicación de recortes, mediante el empleo de instrumentos considerados absolutos incluso desde un punto de vista de gestión. En educación, la dirección, entendida como actividad de gestión, no comparte los mismos valores con las personas que la ejercen: se usa con frecuencia el término "monitorear" para evitar un vocabulario de gestión con frecuencia calificado de "neoliberal”. (BARRÈRE, 2006) Muchas de estas personas no hacen una distinción clara entre "control" y "evaluación", incluso si las prácticas de auditoría en los centros educativos son actualmente desarrolladas por los cuerpos de inspección. (POWER, 1997)

\section{Una descentralización limitada en términos de transferencia de responsabilidades}

La descentralización tiene un alcance limitado. Sin duda, las primeras leyes descentralizadoras delegaron importantes poderes en autoridades locales en cuanto a la construcción, renovación y equipamiento de los centros educativos. Estas últimas hicieron uso de las nuevas responsabilidades para la obtención de inversiones significativas y algunas prestigiosas operaciones con propósitos electorales. El objetivo no era otro que probar que las autoridades locales podían hacer un mejor trabajo en un contexto de inversión reducida desde el Estado. Sin embargo, en educación, la descentralización fue frenada a inicios de los 1980. No fue hasta 1995 cuando una nueva ley transfirió las responsabilidades en formación profesional desde el Estado hasta las Autoridades Regionales. En cualquier caso, esta descentralización fue sólo parcial: el Estado sigue gestionando los centros de formación profesional y escuelas de aprendices, llevándolo a cabo incluso en aquellas regiones entre cuyas competencias se incluye la provisión de formación profesional mediante planes quinquenales.

La educación permanece centralizada y bajo el estrecho control del Estado. El Estado se encuentra a cargo de la definición del currículum, de la carga lectiva, de la selección, contratación y trayectoria profesional del profesorado y otro tipo de personal, de la formación inicial y continua de estos, del control y la inspección de los centros, de la orientación e inserción profesional del alumnado, de los diplomas, certificaciones y reconocimiento de cualificaciones. Si la transferencia fue implementada en el sistema educativo dando más autonomía a los rectores, estos permanecen todavía muy dependientes de las decisiones tomadas por el Ministerio de Educación. En las regiones, las relaciones entre 
Estado y autoridades locales pueden ser tensas debido a conflictos derivados de compartir jurisdicción, o a la oposición ideológica. De hecho, los sectores de educación primaria y secundaria se encuentran cómodamente emparejados desde una perspectiva cultural e institucional, y ello no facilita ni la cooperación ni la gobernanza compartida. Los contratos basados en objetivos definen relaciones entre el Estado y las autoridades locales, pero también entre las autoridades locales de educación (Rectorados) y los centros educativos. Algunas redes conformadas por centros educativos están emergiendo fundamentalmente para superar la gran división entre los sectores de educación primaria y secundaria, y para desarrollar la cooperación en torno a la implementación de un marco de habilidades básicas.

El flujo de funcionarios a las autoridades locales, llevado a la práctica por personal técnico y de mantenimiento en los centros educativos, es motivo de un intenso debate. Varios intentos similares fueron realizados con consejeros de orientación escolar. Pero éstos fracasaron debido a las protestas a gran escala realizadas por el cuerpo profesional de los Consejos de Orientación Psicológica (Conseils d'Orientation Psychologues), ideológicamente contrario a un concepto de asesoramiento defendido por las autoridades locales en sólida cooperación con intereses de negocio regionales y servicios implicados en la evaluación de habilidades o en la inserción profesional. A modo de lobby interno, el asesoramiento presenta una poderosa influencia tanto sobre el Ministerio de Educación como en forma de leyes. Expertos y diseñadores de políticas reflexionan en la actualidad en torno a la creación de un servicio de asesoramiento público regional, pero por el momento no ha sido realizada una propuesta concreta por parte del Ministerio.

\section{La retención de una tradición cultural a pesar de una política de habilidades básicas}

El Marco Común de Conocimientos y Competencias (Socle Commun de Connaissances et de Compétences) es la obra maestra de la Ley de Educación 2005, aprobada bajo la administración del ministro François Fillon. El socle acumulaba un completo juego de narrativas (podríamos decir, incluso, storytelling) que le servían para presentarse como el legado de sucesivos planes educativos desde la fundación del Sistema Escolar Republicano. Sin embargo, como se ha visto, este marco es una traducción, con algunos cambios menores, del Marco Europeo de Competencias Clave diseñado en 2004 por la Comisión Europea, cuando Francia se implicó por varios años en la implementación de la Estrategia de Lisboa. Éste sólo resumía, después de más de dos décadas, la itinerante política de competencias implementada en Estados Unidos y Reino Unido a principios de los 80. (OZGA; JONES, 2006) En cualquier caso, el Marco Común de Conocimientos y Competencias francés se encuentra completamente desconectado de las cuestiones de evaluación y de aprendizaje. Éste ha conducido a una auténtica guerra 
curricular de batallas ideológicas fuertemente mediatizadas. (SHOR, 1986) En términos de currículum, los modernizadores son lo contrario a los tradicionalistas. Los primeros desean adaptar la enseñanza de materias escolares a las necesidades del alumnado, demandando un vínculo más fuerte entre contenidos a transmitir por el profesorado y habilidades a ser adquiridas por el alumnado. Por su parte, los segundos pretenden mantener el requerimiento de un alto nivel de contenidos, al tiempo que critican una concepción instrumental del currículum que distorsiona la cultura transmitida al alumnado. Ésa es la razón por la que el actual gobierno socialista, atendiendo las demandas del principal sindicato de profesores, ha incorporado la cultura general al marco básico de competencias y saberes. De forma independiente, esta división se sitúa más allá de la tradicional oposición entre izquierda y derecha.

Desde esta perspectiva, la acción del Estado se encuentra dividida entre varios requerimientos contradictorios. Por un lado, aboga por un marco de competencias como herramienta de diversificación pedagógica para el sostenimiento de la orientación individualizada y el logro académico del alumnado. Pero, al mismo tiempo, permanece unida a un objetivo de igual condición de enseñanza para cada miembro del mismo y defiende una concepción estandarizada del currículum. Además de esta paradoja entre estandarización y diversificación, existe una fuerte tensión entre evaluación y currículum.

Cada miembro del profesorado es considerado autónomo en su aula, y en nombre de esta "libertad pedagógica" es reconocido y reafirmado el Código Pedagógico. Al mismo tiempo, como miembros del funcionariado, el profesorado tiene que aplicar las instrucciones oficiales emitidas por el Ministerio para la implementación del currículum. Sin embargo, éstos disponen de un poder discrecional para la evaluación del alumnado por lo general a través de las calificaciones. La ausencia de vínculo entre currículum y evaluaciones impide a los profesores considerar la cuestión de las competencias del alumnado, habida cuenta que éstos y éstas no se sienten interesados por el aprendizaje de los estudiantes sino por el contenido a impartir. Ello explica por qué el Consejo Superior del Currículum (Conseil Supérieur des Programmes) ha tenido que adaptar el currículum al marco de competencias, habiendo propuesto recientemente la implementación de la evaluación sin notas, para la graduación de estudiantes de acuerdo a sus niveles de dificultad de aprendizaje, como ha sido llevado ya a cabo por otros países europeos. Pero hasta el momento, las recomendaciones del Consejo Superior no han tenido demasiado impacto en el diseño de políticas.

\section{Elección de centro sin desarrollo del mercado}

La ideología del mercado fue puesta al servicio de la política desreguladora de zonas de captación. La derecha, bajo el gobierno de Sarkozy, buscaba 
promocionar la cuestión de la elección de centro mientras que la izquierda se oponía con fuerza defendiendo la mixtura social en las escuelas. Sin embargo, al contrario que en Inglaterra, esta desregulación se vinculaba a un cierto número de requerimientos que limitaban su alcance. (BALL, 2008) En primer lugar, se encontraba el problema de las plazas limitadas en los mejores centros. En segundo, la selección e inscripción del alumnado tenía que respetar criterios estrictos (hermanos, becas, necesidades especiales, etc.) que restringían el número de casos examinados desde regulaciones burocráticas, las cuales, a su vez, restringían la voz de los padres y sus posibilidades de movilización. Los directores de centro, junto a algunos responsables locales, se mostraron también reacios a implementar esta política. En cambio, como ha sido observado en otros lugares, el resultado condujo al aumento de la segregación social y al desafío de aquellos centros que perdían a sus mejores estudiantes, además del hecho de que esta política no compensaba la dominación de las familias de clase media y alta en las estrategias de elección de centro. Tampoco tuvo éxito en el desarrollo de un mercado educativo con la correspondiente competición entre centros, como sí sucedió en el Reino Unido. (TOMLINSON, 2005; WALFORD, 2006) Esta política fue abandonada por la izquierda cuando accedió al poder en 2012.

Simultáneamente, el fuerte vínculo a la igualdad de oportunidades ha conducido a la concepción e implementación de algunos sistemas mixtos de elección de centro desde principios meritocráticos de selección que tienden a discriminar a los alumnos más desfavorecidos. Esa es la razón por la que algunas instituciones de educación superior, siguiendo el ejemplo de París Sciences-Po, han desarrollado procedimientos tutelados en centros con dificultades, mientras aulas preparatorias para el acceso a las instituciones de educación superior han abierto sus puertas a los alumnos que lo merecían en nombre de la discriminación positiva. Como algunos resultados de investigaciones sociológicas demuestran, esta acción ha permitido a las instituciones de educación superior mostrar una política de apertura orientada a silenciar las críticas recibidas por su elitismo, este último derivado del mantenimiento de una fuerte selección en las pruebas de acceso. El otro sistema inventado por la derecha se sustentaba en los Polos de Excelencia (Internats d'Excellence), internados escolares que copian el modelo de escuela concertada estadounidense (charter schools). Estos centros proponen a estudiantes desfavorecidos mejor apoyo en enseñanza y aprendizaje, al tiempo que aíslan a cada uno de éstos de su contexto social y familiar para ofrecerles mejores condiciones de estudio. Sin embargo, la gestión de cada centro aparece como extremadamente heterogénea, ya que depende de la implicación de las autoridades locales, de la movilización de equipos docentes, de los procedimientos para la contratación, del grado de autonomía de la estructura pedagógica, etc. Estos centros han alimentado las quejas ante una meritocracia imaginaria, pese a que sirven como herramientas de propaganda en los medios para la promoción de una discriminación positiva con limitados efectos en su tramo final. 


\section{Los fracasos del reformismo conservador de la izquierda}

Cuando los socialistas accedieron al poder en 2012, la política de diversificación y elección de centro escolar fue detenida, pues aquéllos abogaban por la reducción de la desigualdad de oportunidades así como por el fortalecimiento de la escuela mixta. La autonomía escolar, la cual quiso promover la derecha otorgando mayor responsabilidad a los directores de centro, fue igualmente detenida mientras una concepción legitimadora devolvía el poder al Cuerpo de Inspección General. Vincent Peillon, Ministro de la Educación Nacional, reunió a todos los altos directivos de los distritos parisinos, informándoles de que "gestión" y "gobernanza" no pertenecían a su vocabulario. En cambio, una retórica en torno a nuevos fundamentos del sistema escolar fue difundida, al tiempo que los principios del Sistema Escolar Republicano eran reafirmados, particularmente desde la implantación de la enseñanza de la "moral laica" en los centros. En efecto, este ministro formado en filosofía ha permanecido muy unido a los valores y principios republicanos, siendo inspirado por los fundadores del Sistema Escolar Republicano, en especial por Ferdinand Buisson. Apegado a sus valores republicanos, este gobierno de izquierdas está promoviendo el Marco de Competencias como medio para la democratización y para la reducción de la desigualdad de oportunidades.

La Comisión para el Sistema Educativo de Nueva Planta, creada por el Ministro para la implementación de una nueva ley, a pesar de sus numerosos grupos de trabajo y de su cobertura mediática, no ha llevado a cabo una reforma sustancial. La idea de acentuar los esfuerzos del sistema educativo sobre la Educación Primaria ha asumido únicamente algunas de las recomendaciones de las organizaciones internacionales. El desarrollo de un plan nacional para las tecnologías digitales se alinea con propósitos similares al tiempo que no implica al Ministerio, mientras que el equipamiento permanece principalmente dependiente de las autoridades locales. Es más, la reacción de las autoridades locales explica el fracaso de la reforma de los horarios escolares cuando ésta fue presentada como programa clave en el marco de la Ley. Sucumbiendo ante la corporación de médicos cercana a la Academia de las Ciencias, el Ministro decidió implementar un plan nacional para la estructuración de los horarios escolares en educación primaria después de que éste fuera aceptado por los sindicatos. Una vez que la reforma ya había sido anunciada, no pasó mucho tiempo hasta que los propios sindicatos desautorizaran al Ministro, mientras profesorado, autoridades locales y padres expresaban su insatisfacción por una reforma deficientemente preparada, vagamente negociada así como carente de fundamento. Idéntica aceptación encontraron la reforma de los Institutos Universitarios de Formación de Maestros (IUFM) y la de Formación Inicial del Profesorado: fueron rápidamente incrustadas en un torbellino burocrático y propiciaron un resurgimiento de los conflictos de intereses. Mientras tanto, el Ministro intentaba incluir la reforma de la profesión docente 
en la agenda política sin tiempo para implementarla y sin el apoyo suficiente de su círculo. Estos últimos preferían focalizar sus acciones en la reestructuración del currículum nacional de acuerdo al marco de competencias, y en intentar promover la escuela mixta mediante la transformación de la oferta educativa, en especial desde la disminución de ciertas opciones elitistas como alemán, latín o algunos cursos bilingües que habían desembocado en múltiples protestas de parte de sindicatos y grupos de interés en torno a disciplinas concretas.

\section{Conclusión}

Una falta de reestructuración de la profesión docente, una limitada autonomía para las escuelas, un marco para la elección de centro de dimensiones limitadas, un mercado de trabajo restringido, una ideología de gestión con una significativa oposición, una descentralización insatisfactoria: en esas condiciones es complicado defender que el New Public Management haya sido implementado en el sistema educativo francés. Ello contrasta con fuerza con las reformas recientes en el área de sanidad, donde la gestión del desempeño, procedimientos de calidad, flexibilidad y movilidad, contratos y agencias, han creado una nueva configuración del servicio público en los hospitales. Esta diferencia se explica por varias razones: el servicio de sanidad pública en Francia se ha construido con una implicación importante de los colectivos locales, mientras que la intervención del Estado central se ha producido de forma mucho más tardía que en el caso de la educación. Los médicos siempre se han mostrado favorables a las soluciones liberales y a la "managerialización" contra el Estado, al contrario que el cuerpo docente, apegado al Estado central así como a sus principios republicanos. La segmentación entre grupos profesionales en sanidad es mucho más importante que en educación, sobre todo entre médicos y enfermeros, lo que ha hecho disminuir el impacto de los comportamientos solidarios de resistencia y protesta. Finalmente, las asociaciones de enfermos se han mostrado muy activas a partir delas epidemias del sida y de la sangre envenenada en la demanda, junto a las industrias farmacéuticas, de una reorganización de la actividad hospitalaria; en educación, sin embargo, la voz de padres y madres se ha mantenido minoritaria en un marco general marcado por la presencia de grupos de interés ampliamente endógenos respecto al sistema educativo, propiedad que ha favorecido que se neutralicen desde sus propias reivindicaciones.

En educación, sólo la rendición de cuentas administrativa y financiera ha penetrado en las instituciones a largo plazo, sin haber presentado consecuencias importantes en el trabajo del profesorado. Directores e inspectores se encuentran desarrollando evaluaciones y auditorías, integrándolas en contratos, pero la estructura burocrática sigue predominando. Sin embargo, las reflexiones actuales 
entre expertos y responsables de políticas educativas, tanto de derecha como de izquierda, nos llevan a pensar que éstos persiguen una especie de tercera vía entre el Estado y el mercado. Un tercer paso en la descentralización, siguiendo la creación de nuevas entidades regionales y persiguiendo una reestructuración de las formas en que las responsabilidades locales son compartidas, podría ser el objetivo principal de la siguiente reforma educativa. Otra cuestión se encuentra vinculada a la reforma del estatus de los funcionarios, la cual podría tener consecuencias en el servicio público que proporciona la Educación Nacional. En cualquier caso, la confrontación entre izquierda y derecha en relación a este proyecto sigue siendo decisiva, y los sindicatos se encuentran prestos a defender con ferocidad sus derechos.

\section{Referencias bibliográficas}

BALL, S.J. The Education Debate. Bristol: The Policy Press, 2008.

BEZES, P. Réinventer l'État. Les réformes de l'administration française. Paris: PUF, 2009.

CLARKE, J.; NEWMAN, J. The managerial state: power, politics and ideology in the remaking of social welfare. London: Sage, 1997.

CROZIER, M. Etat moderne, Etat modeste. Stratégies pour un autre changement. Paris: Fayard, 1986.

DEROUET, J.-L. École et Justice. De l'égalité des chances aux compromis locaux?. Paris: Métailié, 1992.

DEROUET, J.-L.; DEROUET-BESSON, M.-C. Repenser la justice dans l'éducation et la formation. Genève: Peter Lang, 2008.

DEROUET, J.-L.; NORMAND, R. Caesars and Rubicon. The hesitations of French policymakers in identifying a Third Way in education and training. Journal of Educational Administration and History, n. 43, 2010, p. 97-121.

FRASER, N. Qu'est-ce que la justice sociale? Reconnaissance et redistribution. Paris: La Découverte, 2013.

GEWIRTZ, S. et al. (Eds.). Changing teacher professionalism: International trends, challenges and ways forward. London: Routledge, 2009.

GLEESON, D.; HUSBANDS, C. (Eds.). The Performing School. Managing Learning and Teaching in a Performance Culture. London: Routledge Falmer, 2001.

HENRY, M., et al. The OECD, Globalization, and Education Policy. Oxford: Pergamon-Elsevier, 2001.

HIRSCHMAN, A. The Rethoric of Reaction: Perversity, Futility, Jeopardy. Cambridge: Harvard University Press, 1991. 
HONNETH, A. Le droit de la Liberté: Esquisse d'une ethnicité démocratique. Paris: Gallimard, 2015.

HOOD, C. A public management for all seasons? Public administration, v. 69, n. 1, 1991, p. 3-19.

HOOD, C.; PETERS, G. The middle aging of new public management: into the age of paradox? Journal of public administration research and theory, v. 14, n. 3, 2004, p. 267-282.

LAWN, M.; NORMAND, R. Shaping of European education. Interdisciplinary Approaches. London: Routledge, 2014.

MAHONY, P.; HEXTALL, I. Reconstructing teaching: standards, performance and accountability. London: Routledge, 2000.

NORMAND, R. La mesure de l'école. Une arithmétique politique des inégalités. Berne: Peter Lang, Presses de l'Ecole Normale Supérieure, 2011.

NORMAND, R. French Educators' Uncertainties and Doubts against Changes influenced by Globalization. In: SEDDON, T.; OZGA, J.; LEVIN, J. Globalization and professions. Routledge: World Yearbook of Education, 2012.

OZGA, J.; JONES, R. Travelling and embedded policy: the case of knowledge transfer. Journal of Education Policy, v. 21, n. 1, 2006, p. 1-19.

POLLITT, C. Managerialism and the Public Services: The Anglo-American Experience. Oxford: Blackwell, 1990.

POLLITT, C.; BOUCKAERT, G. Public Management Reform: A Comparative Analysis. 2. ed. Oxford: Oxford University Press, 2004.

POWER, M. The Audit Society. London: Demos, 1997.

SHOR, I. Culture wars: school and society in the conservative restoration, 1969-1984. Boston: Routledge \& K. Paul, 1986.

TOMLINSON, S. Education in a post-Welfare Society. 2. ed. Nueva York: Open University Press, 2005.

WALFORD, G. Education and the Labour Government: an Evaluation of Two Term. London: Routledge, 2006.

Recebido em 29 de julho de 2015.

Aprovado em 26 de agosto de 2015.

DOI: http://dx.doi.org/10.1590/ES0101-73302015152583 